\section{A Highly Sensitive Phase-Contrast Refractometer for Liquids and Gases}

THE accompanying diagram shows the essential parts of a refractometer using the principle of phase contrast, which is new for this class of instrument. It gives relative measurements, like most of the applica. tions of the Jamin and the Rayleigh refractometers depending on interference. The liquid or gas to be measured is enclosed in the central horizontal cell $C$ about $1 \mathrm{~mm}$. in height, the reference liquid or gas filling the upper and lower portions. The planeparallel frame forming the middle of the cell, and made of glass, plastic or stainless steel with its plane sides lapped, is made tightly adherent to the two highly polished plano-convex lenses of selected homogeneity.

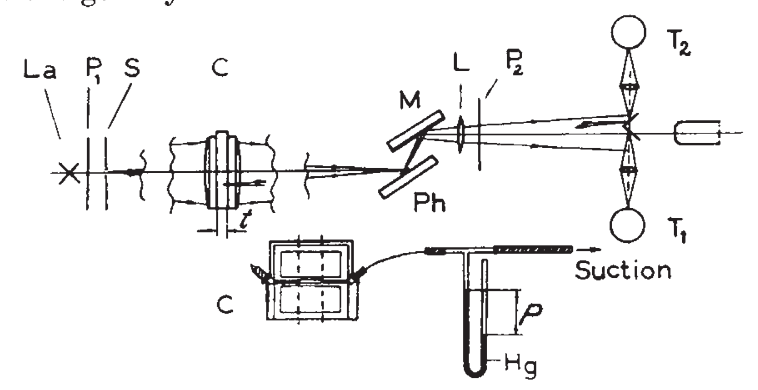

The optical system is symmetrical about this lenslike cell. An image of the entrance slit $S$, illuminated by monochromatic $5461 \mathrm{~A}$. light from a mercury lamp, is formed by this cell on the central part of the phase mirror $P h$. Then the light is passed, by means of the mirror $M$, through the lens $L$, which forms, in the plane dotted in the diagram, the phasecontrast image of the cell $C$. This image can be viewed by an eyepiece or a microscope. But in order to measure the intensity of a defined part of the image of the central cell, compared with the intensity of a second defined part of the image field, these two parts are projected on to the two photomultiplier tubes $T_{1}$ and $T_{2}$, respectively.

The phase mirror is based on the principle described by Françon and Nomarski ${ }^{1}$ and modified by $\mathrm{me}^{2}$. To render the instrument uniformly sensitive over the whole region of measurement, it consists of several portions giving different phase-turning angles. Two 'Polaroids' $P_{2}$ and $P_{2}$ serve to determine and vary the vector origin.

According to the theory previously given ${ }^{3,4}$, the difference of refractive index between the two liquids, $n_{2}-n_{1}$, is determined by the intensity ratio between the two fields. This is a function of the path difference or, more exactly, of the phase difference angle $\left(n_{2}-n_{1}\right) t .2 \pi / \lambda$. It may be an arbitrary part of a complete turn, or several turns, but full details of the procedures eannot be given here, only mentioning that the number of whole turns is counted as the displacement of the zero order fringe from a double slit system as used in Young's experiment.

A very simple experiment is to make the optical path difference exactly one wave-length, that is, the phase angle exactly $2 \pi$, since it can be done visually by equalizing the intensities as in a Lummer-Brodhun photometer. The air of the central cell is pumped out, and the positions of the manometer corresponding to equal field intensities are noted. The difference of pressure equivalent to one wave-length was found to be $149 \mathrm{~mm}$. of mercury; the thickness $t$ being
$10.4 \mathrm{~mm}$. and the air temperature $22 \cdot 4^{\circ} \mathrm{C}$. This gives a refractive index of $2.68 \times 10^{-4}$ for air of the same temperature and $760 \mathrm{~mm}$. mercury pressure. When reduced to $20 \cdot 0^{\circ} \mathrm{C}$., the result is $2 \cdot 70 \times 10^{-4}$, which, considering the very simple means used for making the measurements, is in good accordance with the precision measurements of $2.731 \times 10^{-45}$.

In this Laboratory we measure photoelectrically with an accuracy of 0.5 per cent per amplitude in a phase-contrast field. As the vector is about unit length, the accuracy approximates to $0.5 \times 10^{-2} / 2 \pi$ of a turn, or for the refractive index difference at $t \times 10$ $\mathrm{mm}$., which thickness contains 18,300 periods, the precision is $5 \times 10^{-8}$. This is mora than ten times better than the results obtained by any interfero. meter $^{5}$. An amount of at least $0.1 \mathrm{~cm} .{ }^{3}$ of the liquid under examination is necessary and about $10 \mathrm{~cm} .^{3}$ of reference liquid. For a mixture of heavy and. ordinary water, this means an accuracy of analysis of 0.001 mole per cent. Measurements have not yet been made on account of the lack of precise reference standards.

The precision can be reduced by making $t$ smaller, and it can probably be raised by increasing it to some centimetres. This is possible with maintained focusing of the image, as the focal lengths must be rather long for other reasons. The temperature remains fairly well constant because the measuring cell is surrounded by the reference liquid and separated from it by thin walls.

I am now working with Mr. Lars Johansson, chemical engineer, to elaborate the apparatus for routine use.

ERIK Ingelstam

Optics Laboratory,

Royal Institute of Technology, Stockholm 70 .

July 3.

${ }^{1}$ Francon, M., and Nomarski, (4., C.R. Acad. Sci., Paris, 230, 1392 (1950).

Ingelstam, E., Ark. Fys., 3, No. 2 (1951).

${ }^{3}$ Ingelstam, E., Ark. Fys., 3, No. 20 (1951).

Ingelstam, E., Colloque intern. sur le contraste de phase et le contraste par interférence, Paris, May 1951 (in the press).

5 See, for example, Candler, C., "Modern Interferometers" (Iondon, 1951).

\section{Fly Reactions to Insecticidal Deposits: a New Test Technique}

Mosis laboratory methods for evaluating residual insecticides involve crowding a number of flies together in rather small containers. Such crowding stimulates activity, which may cause the flies to pick up abnormal amounts of insecticide. These conditions are unnatural, and while useful and reproducible results may be obtained, their relevance to field performance is doubtful.

We have found that the difficulties can be overcome by using a 'single fly' technique, based on the exposure in a large treatment cage of one fly at a time. Interference from other flies is avoided and fly behaviour can be studied in detail. Three-day-old well-fed female houseflies, which, if undisturbed, normally remain immobile on an untreated surface for three to five hours at $24-26^{\circ} \mathrm{C}$., were used in all tests.

A cubical cage of 2 -ft. side forms the container, and is about the minimum size in which a fly shows no reaction to confinement. The cage consists of a light wooden frame having a paper back as the treat- 